\title{
Safety Evaluation of the Aqueous Extract Kothala Himbutu (Salacia reticulata) Stem in the Hepatic Gene Expression Profile of Normal Mice Using DNA Microarrays
}

\author{
Ryanghyok Im, Hiroshi Mano, Sachie Nakatani, Jun Shimizu, and Masahiro Wada ${ }^{\dagger}$ \\ Department of Clinical Dietetics and Human Nutrition, Faculty of Pharmaceutical Sciences, Josai University, \\ 1-1 Keyakidai, Sakado, Saitama 350-0295, Japan
}

Received November 15, 2007; Accepted August 19, 2008; Online Publication, December 7, 2008

[doi:10.1271/bbb.70745]

\begin{abstract}
Kothala himbutu is a traditional Ayurvedic medicinal plant used to treat diabetes. We aimed to evaluate the safety of an aqueous extract of Kothala himbutu stem (KTE) in normal mice. The mice were divided into two groups: one was administered KTE and the other distilled water for 3 weeks. During the test period, the groups showed no significant differences in body weight gain or plasma parameters, such as fasting blood glucose level, oral glucose tolerance test, or aspartate transaminase (AST) or alanine transaminase (ALT) activity. DNA microarray analysis revealed that expression of genes of known function, such as those for the stress response, ribosomal proteins, transcription, cell function, the inflammatory/immune response, and metabolism (xenobiotic, glutathione, etc.) remained largely unaffected by KTE. However some genes such as catechol-o-methyltransferase and succinyl-CoA synthetase were regulated by KTE, indicating that KTE is not toxic to normal mice and might be effective as a functional food.
\end{abstract}

Key words: Salacia reticulata; DNA microarray; food safety; C57BL/6J mice

Salacia reticulata (Hippocrateaceae), referred to as Kothala himbutu (KT) in Singhalese, is a woody climber native to Sri Lanka, where it is a well-known traditional medicinal plant. The aqueous extract of the roots and stems of this plant is extensively used in Ayurvedic medicine in India and Sri Lanka in treatment in the initial stages of diabetes. ${ }^{1,2)}$ It reduces fasting blood glucose levels in rats and mild type-2 diabetic patients. ${ }^{3,4)}$ Controlling the fasting blood glucose level is a critical step in the treatment of type- 2 diabetes, but little is known about the mechanism behind this effect.

Previous studies in our laboratory using a diabetic model of KK-Ay mice have revealed the mechanisms by which the aqueous extract of the Kothala himbutu stem
(KTE) decreases fasting blood glucose. An active compound in KTE, mangiferin, acts directly on liver cells and downregulates the gluconeogenic pathway by regulating fructose-1,6-bisphosphatase expression, resulting in lowered fasting blood glucose levels in the diabetic patient. ${ }^{5)}$

The recent concept of functional food, intermediates between food and medicine, has gained considerable attention as a key concept in the global food industry. ${ }^{6,7)}$ As a result, many people in the United States, Japan, and other industrialized countries now use plants as functional foods in the form of herbal teas and dietary supplements to prevent disease and maintain good health. KT is available commercially as a dietary supplement for the prevention of diabetes and obesity in countries such as the United States and Japan.

An assessment of the safety of KTE, including tests for oral toxicity and chromosomal aberrations in Sprague-Dawley rats, has been performed. ${ }^{8)}$ Only limited information is available on the safety of KTE, while no gene expression profile of the effects of KTE in normal mice has been reported yet.

There are various methods, including in vitro and in vivo studies, of evaluating food safety. ${ }^{9)}$ Nutrigenomics, which utilizes high through-put analysis tools, has emerged as an immensely valuable strategy in nutrition research. ${ }^{10)}$ DNA microarray technology is one such tool useful in the evaluation of the safety of foods and natural products. ${ }^{11,12)}$ Hence, we investigated the effects of KTE on gene expression in the liver of normal mice using DNA microarrays carrying approximately 10,000 known mouse genes.

The purpose of this study was to evaluate the safety of KTE consumption by non-diabetic mice with a view to validating its presumed benefits in healthy and prediabetic people for its supposed preventive effect. The safety of KTE consumption in normal C57BL/6J mice was evaluated by examining plasma parameters, includ-

\footnotetext{
$\dagger$ To whom correspondence should be addressed. Fax: +81-49-271-7240; E-mail: mwada@josai.ac.jp

Abbreviations: CYP, cytochrome P450; GST, glutathione S-transferase; KT, Kothala himbutu; KTE, aqueous extract of the stem of Kothala himbutu; KTED, freeze-dried aqueous extract of the stem of Kothala himbutu
} 
ing postprandial and fasting blood glucose levels, AST and ALT activities, and the hepatic gene expression profile.

\section{Materials and Methods}

Preparation of aqueous extract of KT. Whole stems (excluding leaves and roots) of KT, harvested in Sri Lanka, were purchased from the Ayurveda Kothalahimbutu Association (Tokyo). The plant was identified by Dr. G. A. S. Premakumara, Herbal Technology Section, Industrial Technology Institute (Colombo, Sri Lanka). A voucher specimen of the stems was deposited in our laboratory (Department of Clinical Dietetics and Human Nutrition, Faculty of Pharmaceutical Sciences, Josai University, Japan). The stems were dried and ground to a fine powder. One hundred $g$ of the powder was boiled in 12.5 liters of distilled water for $2 \mathrm{~h}$. The solvent was filtered and centrifuged for $10 \mathrm{~min}$ at $3,000 \times g$ to remove particulate matter. The supernatant was designated KT aqueous extract (KTE). KTE was freeze dried (Freeze Dryer System, Labconco, Kansas City, MO) yielding the KTED $(9 \mathrm{~g})$. The yield $(\mathrm{w} / \mathrm{w})$ of KTED was about $3.8 \%$. The amount of KTED obtained from KTE was about $0.45 \mathrm{mg}$ of dry matter $/ \mathrm{ml}$.

Animals and diet. We purchased 12 male 4-week-old C57BL/6J mice from Clea Japan (Tokyo). The mice were housed individually in an environmentally controlled room under constant temperature $\left(25 \pm 3{ }^{\circ} \mathrm{C}\right)$ and a 12-h light/dark cycle. Experiments were performed on the mice after an acclimatization period of 1 week.

Under standardized conditions, the mice (5 weeks old at that time) were divided into two groups. One group was treated with KTE, and the other (the control) was administered distilled water. The treatment and control groups were given with free access to KTE and distilled water respectively. On an average, the mice in the treatment group consumed approximately $2.3 \mathrm{mg}$ of dry matter $/ 5 \mathrm{ml}$ of KTE per d, whereas those in the control group drank approximately $5 \mathrm{ml}$ of distilled water per $\mathrm{d}$. Both groups were given free access to a standard diet (MF diet, Oriental Yeast, Tokyo) for 3 weeks.

The mice were euthanized by cervical dislocation at the age of 8 weeks after overnight fasting. They were then dissected, and the liver tissue was harvested for total RNA isolation. Animal care was in conformance with "Standards Relating to the Care and Management of Experimental Animals" laid down by the Ministry of Education of Japan and the Institutional Animal Care and Use Committee of Josai University.

Fasting blood glucose levels and oral glucose tolerance test. The fasting blood glucose levels of the mice the were examined after fasting them for $10 \mathrm{~h}$. The blood samples were collected from the tail vein. The blood glucose concentration was measured using a Glucocard meter (Arkray, Kyoto).
The oral glucose tolerance test (OGTT) was performed on overnight-fasted mice. The control and KTEtreated mice were administered $200 \mu \mathrm{l}$ of distilled water and $200 \mu \mathrm{l}$ of KTE respectively, followed by $2 \mathrm{~g} / \mathrm{kg}$ of glucose. Blood was collected from the tail vein immediately before and 30, 60, and $120 \mathrm{~min}$ after administration of glucose to determine blood glucose levels.

Biochemical analysis of plasma. The plasma concentration of glucose was measured with a Glucose C II test Wako kit (Wako Pure Chemicals, Tokyo). Alanine aminotransferase (ALT, EC1.1.1.27) activity and aspartate aminotransferase (AST, EC2.6.1.1) activity were measured using a Transamilase C-II test Wako kit (Wako Pure Chemicals, Tokyo).

Preparation of RNA. Total RNA was isolated using Trizol reagent (Invitrogen, Tokyo) according to the manufacturer's instructions. It was dissolved in diethyl pyrocarbonate-treated distilled water. The concentration of total RNA was estimated from the absorbance at $260 \mathrm{~nm}$. RNAs from the liver tissue were used for DNA microarray analysis.

DNA microarray analysis. All experiments and analyses were performed according to the protocol in the GeneChip Expression Analysis Technical Manual (Affymetrix). Equal amounts of total RNA from six mice in each group were pooled. The mRNAs were reverse-transcribed with $\mathrm{T} 7-(\mathrm{dT})_{24}$ primer and copied into double-strand cDNAs (Superscript Choice System, Invitrogen). Biotin-labeled cRNAs were synthesized (High Yield RNA Transcript Labeling Kit, Affymetrix) and fragmented by heating at $94^{\circ} \mathrm{C}$ for $35 \mathrm{~min}$. Fragmented cRNAs were hybridized with a GeneChip Murine Genome U74A Array (Affymetrix) according to the Affymetrix protocol. After hybridization and subsequent washing and staining using Affymetrix Fluidics Station 400, the arrays were scanned using an Affymetrix array scanner, and the fluorescence intensity was measured using Microarray Suite 5.0 (Affymetrix).

Statistical analysis. Data were expressed as means \pm standard deviation (SD). Treatment effects were analyzed by the Student's $t$-test. Differences with $P<0.05$ were considered statistically significant. Expression analysis systemic explorer (EASE) analysis ${ }^{13)}$ was carried out using the Database for Annotation, Visualization, and Integrated Discovery (DAVID). ${ }^{14)}$

\section{Results}

Body weight gain, liver weight, fasting blood glucose levels, and plasma parameters

Food intake during the 3-week experimental period did not differ significantly between the two groups. The body weights of the mice in both groups increased 
Table 1. Body Weight Gain, Liver Weight, Fasting Blood Glucose, and Plasma Parameters at the End of the Experimental Period

\begin{tabular}{lcc}
\hline \hline & Control & KTE \\
\hline Body weight gain (3 weeks) & $5.1 \pm 1.1$ & $4.3 \pm 1.4$ \\
Liver weight & $5.64 \pm 0.53$ & $5.29 \pm 0.39$ \\
$\quad$ (g/100g body weight) & & \\
Fasting blood glucose (mg/dl) & $96.7 \pm 4.2$ & $91.7 \pm 1.2$ \\
Plasma & & \\
AST (U/I) & $32.2 \pm 4.2$ & $34.4 \pm 9.0$ \\
ALT (U/1) & $14.1 \pm 2.1$ & $14.0 \pm 5.0$ \\
\hline
\end{tabular}

Data are as mean $\pm \mathrm{SD}, n=6-8$.

AST, asparate aminotransferase; ALT, alanine aminotransferase

steadily during the experimental period. At the end of study, there was no significant difference between the body weight gains of the KTE-treated mice and the control mice. The liver weight ( $\mathrm{g} / 100 \mathrm{~g}$ of body weight) did not significantly differ between the two groups. As compared to the control mice, the KTE-treated mice tended to have decreased fasting blood glucose levels and plasma glucose concentrations, but the differences between the groups were not significant. Moreover, there were no significant differences in aspartate aminotransferase (AST) activity or alanine aminotransferase (ALT) activity between the KTE-treated and the control mice (Table 1).

\section{Oral glucose tolerance test}

The influence of KTE on postprandial blood glucose levels was examined in glucose-loaded mice. There was no significant difference in the postprandial blood glucose levels between the KTE-treated and the control mice (Fig. 1).

DNA microarray analysis of liver tissue of KTEtreated mice

Changes in gene expression profiles in the liver cells of the treatment and control groups were investigated by DNA microarrays (Table 2). A total of 5,808 genes were identified as present in both groups by Microarray Suite 5.0 (Affymetrix) on the basis of expression profiles. These genes were then classified into 10 groups on the basis of their known biological functions: transcription (818), cell function (705), inflammatory/immune response (110), metabolism (1403), angiogenesis (59), transport (814), stress response (39), ribosomal proteins (34), and other (522). Genes to which a function could not be ascribed were classified as unknown $(1,363)$. We found that 207 genes were upregulated $(n=194)$ or downregulated $(n=13)$ by 2 -fold or more in the KTEtreated mice as compared with the control mice. The EASE score (a Fisher Exact Statistic) was used to indicate the probability that a set of genes was present by sampling of annotated genes on the GeneChip Murine Genome U74A Array. Analysis of EASE score was carried out using web-accessible tools available in DAVID on the National Institute of Allergy and Infectious Disease (NIAID) website, as described

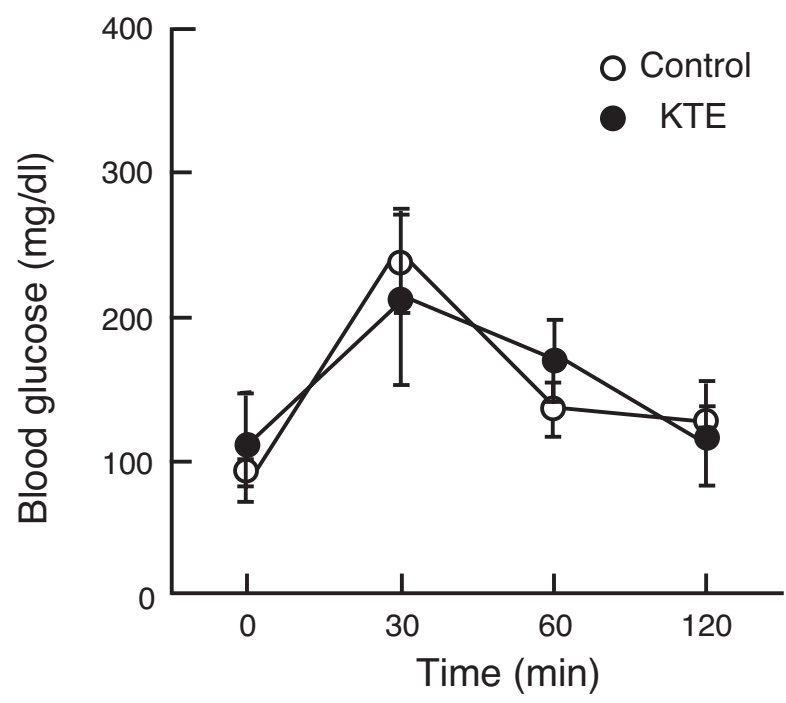

Fig. 1. Influence of KTE on Blood Glucose Levels during the Oral Glucose Tolerance Test (OGTT).

OGTT was performed on C57BL/6J mice administered KTE or distilled water for drinking for 4 weeks. After $16 \mathrm{~h}$ of fasting, glucose $(2 \mathrm{~g} / \mathrm{kg})$ was administered orally. Before the administration of glucose, the control mice were administered $200 \mu \mathrm{l}$ of distilled water, and the KTE treated mice were administered $200 \mu \mathrm{l}$ of KTE. Blood samples were collected before and at 30, 60, and $120 \mathrm{~min}$ after glucose administration. Each point represents the mean $\pm \mathrm{SD}$ $(n=6-8)$.

previously. ${ }^{13,14)}$ The EASE scores of all categories were determined to be greater than 0.05 (Table 2).

CYP51, CYP2C70, and CYP2A5 were upregulated and CYP 7B1 was downregulated by KTE. Additionally, glutachione S-transferases (GSTA2, GSTA4, GSTT1, GSTM2, GSTM5, and GSTM6) were also upregulated by KTE (Table 2). Moreover, the genes for some enzymes involved in the energy metabolic process exhibited changes in expression: catechol-o-methyltransferase and monoamine oxidase A (the enzyme that degrades catecholamines), and succinyl-CoA synthetase and 3-hydroxy-3-methylglutaryl-CoA reductase were upregulated, and acyl-CoA synthetase medium-chain family member 3 was downregulated by KTE (Table 2).

\section{Discussion}

The metabolic syndrome, comprising obesity, insulin resistance, and dyslipidemia, has become a worldwide epidemic of type-2 insulin-resistant diabetes mellitus. ${ }^{15)}$ KTE is used as an Ayurvedic dietary supplement in United States and Japan for the prevention of metabolic syndrome, especially diabetes. In this study, we focused on evaluating the safety of KTE consumption in nondiabetic mice, with a view to validating its presumed benefits to healthy people consuming KTE as a functional food.

There was no significant difference in food intake or body weight gain between the KTE-treated and the control mice during the experimental period. The values 
Table 2. Gene Expression in C57BL/6J Mouse Livers Treated with KTE

\begin{tabular}{|c|c|c|c|}
\hline Affymetrix ID & Gene & KTE/Control ratio & EASE score \\
\hline Transcription (818) & & & 0.760 \\
\hline \multicolumn{4}{|l|}{ Upregulated } \\
\hline 92554_at & C-terminal binding protein 2 & 2.31 & \\
\hline 93802_at & metadherin & 2.22 & \\
\hline 94292_at & serine/threonine kinase receptor associated protein & 2.03 & \\
\hline 98951_at & transcription factor 25 & 2.14 & \\
\hline 97506_at & ring finger protein 2 & 2.06 & \\
\hline 101529_g_at & transcription elongation factor A (SII) 1 & 2.11 & \\
\hline 102062_at & SWI/SNF related, matrix associated, actin dependent regulator of chromatin & 2.16 & \\
\hline 102344_s_at & transcription elongation factor A (SII) 3 & 2.56 & \\
\hline 103073_i_at & TAF9 RNA polymerase II, TATA box binding protein (TBP)-associated fact & 2.20 & \\
\hline 103668_at & suppressor of Ty 6 homolog & 2.29 & \\
\hline 97974_at & zinc finger protein, multitype 1 & 2.05 & \\
\hline 99076_at & nuclear receptor subfamily 1 , group $\mathrm{D}$, member 2 & 2.85 & \\
\hline 101465_at & signal transducer and activator of transcription 1 & 3.26 & \\
\hline 104010_at & zinc finger with KRAB and SCAN domains 14 & 2.06 & \\
\hline 93006_at & nuclear factor $\mathrm{I} / \mathrm{C}$ & 2.02 & \\
\hline 93164_at & ring finger protein 2 & 2.25 & \\
\hline 97185_at & aryl hydrocarbon receptor nuclear translocator & 2.25 & \\
\hline 102144_f_at & splicing factor proline/glutamine rich & 2.33 & \\
\hline 160117_at & thyrotroph embryonic factor & 2.53 & \\
\hline 160377_at & TAR DNA binding protein & 2.24 & \\
\hline 160495_at & aryl-hydrocarbon receptor & 2.02 & \\
\hline 160681_at & poly (A) polymerase alpha & 2.36 & \\
\hline 160941_at & phosphodiesterase $8 \mathrm{~A}$ & 2.23 & \\
\hline 160979_at & C-terminal binding protein 2 & 2.56 & \\
\hline 161562_f_at & zinc finger protein 787 & 2.43 & \\
\hline 93829_at & ROD1 regulator of differentiation 1 & 2.19 & \\
\hline 160663_at & DEAD (Asp-Glu-Ala-Asp) box polypeptide 41 & 2.04 & \\
\hline 160722_at & RNA methyltransferase like 1 & 2.06 & \\
\hline \multicolumn{4}{|l|}{ Downregulated } \\
\hline 94480_at & DNA segment, Chr 1, ERATO Doi 161 & 0.28 & \\
\hline Cell function (705) & & & 0.83 \\
\hline \multicolumn{4}{|l|}{ Upregulated } \\
\hline 98948_at & guanine nucleotide binding protein-like 3 & 2.28 & \\
\hline 97506_at & ring finger protein 2 & 2.06 & \\
\hline 94338_g_at & growth arrest specific 2 & 2.40 & \\
\hline 93164_at & ring finger protein 2 & 2.25 & \\
\hline 160843_at & spindlin 1 & 2.72 & \\
\hline 93764_at & NADH dehydrogenase (ubiquinone) 1 & 2.06 & \\
\hline 94524_at & death associated protein 3 & 3.19 & \\
\hline 97519_at & secreted phosphoprotein 1 & 2.25 & \\
\hline 94338_g_at & growth arrest specific 2 & 2.40 & \\
\hline 95030_at & prolactin receptor & 2.05 & \\
\hline 96753_at & B-cell CLL/lymphoma 7C & 2.07 & \\
\hline 102921_s_at & fas (TNF receptor superfamily member) & 2.06 & \\
\hline 103446_at & interferon induced with helicase $\mathrm{C}$ domain 1 & 2.27 & \\
\hline 104157_at & FAST kinase domains 5 & 3.21 & \\
\hline 100033_at & mutS homolog 2 & 2.44 & \\
\hline 92596_at & calcyclin binding protein & 2.17 & \\
\hline 96104_at & Ring finger protein 145 & 2.47 & \\
\hline 98635_at & ubiquitin-conjugating enzyme E2Z & 2.14 & \\
\hline 104037_at & RWD domain containing 4A & 2.10 & \\
\hline 98972_at & ubiquitin specific peptidase 8 & 2.02 & \\
\hline 99990_at & retinoblastoma binding protein 6 & 2.02 & \\
\hline 99669_at & lectin, galactose binding, soluble 1 & 2.14 & \\
\hline 99062_at & poliovirus receptor-related 3 & 2.52 & \\
\hline 103506_f_at & desmocollin 2 & 2.76 & \\
\hline 97288_at & PDZ domain containing 1 & 2.06 & \\
\hline 98946_at & WD repeat and SOCS box-containing 1 & 2.41 & \\
\hline \multicolumn{4}{|l|}{ Downregulated } \\
\hline 98067_at & cyclin-dependent kinase inhibitor $1 \mathrm{~A}$ & 0.28 & \\
\hline 102016_at & cell death-inducing DFFA-like effector & 0.26 & \\
\hline
\end{tabular}


Table 2. Continued

\begin{tabular}{|c|c|c|c|}
\hline Affymetrix ID & Gene & KTE/Control ratio & EASE score \\
\hline \multicolumn{3}{|c|}{ Inflammatory/Immune response (110) } & 0.188 \\
\hline \multicolumn{4}{|l|}{ Upregulated } \\
\hline 103617_at & CD55 antigen & 2.02 & \\
\hline 92222_f_at & histocompatibility $2, \mathrm{Q}$ region locus 1 & 2.16 & \\
\hline \multicolumn{3}{|c|}{ Metabolism (Xenobiotic, Glutathion, Carbohydrate, Lipid, Catecholamin, etc.) (1403) } & 0.51 \\
\hline \multicolumn{4}{|l|}{ Upregulated } \\
\hline 94916_at & cytochrome P450, family 51 & 2.43 & \\
\hline 95043_at & cytochrome P450, family 2 , subfamily c, polypeptide 70 & 3.01 & \\
\hline 102847_s_at & cytochrome P450, family 2 , subfamily a, polypeptide 5 & 3.66 & \\
\hline 93009 at & glutathione $\mathrm{S}$-transferase, mu 2 & 2.78 & \\
\hline 96085_at & glutathione S-transferase, alpha 4 & 5.09 & \\
\hline 96258_at & microsomal glutathione $S$-transferase 3 & 5.30 & \\
\hline 100042_at & hydroxyacyl glutathione hydrolase & 2.17 & \\
\hline 100629_at & glutathione S-transferase, mu 5 & 2.89 & \\
\hline 95019_at & glutathione S-transferase, theta 1 & 2.13 & \\
\hline 101872_at & glutathione S-transferase, alpha 2 & 4.20 & \\
\hline 104637_at & glutathione S-transferase, mu 6 & 2.38 & \\
\hline 96789_i_at & galactose mutarotase & 2.49 & \\
\hline 96803_at & glucan (1,4-alpha-), branching enzyme 1 & 2.57 & \\
\hline 161594_f_at & galactose mutarotase & 2.29 & \\
\hline 162031_f_at & galactose mutarotase & 2.13 & \\
\hline 160428_at & succinyl-CoA synthetase & 2.00 & \\
\hline 96627_at & phenylalkylamine $\mathrm{Ca} 2+$ antagonist (emopamil) binding protein & 2.41 & \\
\hline 102769_f_at & sterol-C5-desaturase (fungal ERG3, delta-5-desaturase) homolog & 2.63 & \\
\hline 103665_at & ELOVL family member 6 , elongation of long chain fatty acids & 3.42 & \\
\hline 104285_at & 3-hydroxy-3-methylglutaryl-Coenzyme A reductase & 2.28 & \\
\hline 101945_g_at & lysophospholipase 1 & 2.27 & \\
\hline 160388_at & sterol-C4-methyl oxidase-like & 3.66 & \\
\hline 160737_at & lanosterol synthase & 2.05 & \\
\hline 93542_at & phosphotriesterase related & 2.17 & \\
\hline 93749_at & monoamine oxidase $\mathrm{A}$ & 2.09 & \\
\hline 98535_at & catechol-O-methyltransferase & 2.02 & \\
\hline 94380_at & insulin degrading enzyme & 2.02 & \\
\hline 102938_at & leukocyte cell-derived chemotaxin 2 & 2.03 & \\
\hline 160934_s_at & SH3-domain GRB2-like (endophilin) interacting protein 1 & 2.07 & \\
\hline 102017_at & PRP4 pre-mRNA processing factor 4 homolog B & 2.19 & \\
\hline 99960_at & mitogen activated protein kinase kinase 4 & 2.10 & \\
\hline 92192_s_at & proprotein convertase subtilisin/kexin type 5 & 2.01 & \\
\hline 92767_at & bone morphogenetic protein receptor, type $1 \mathrm{~A}$ & 2.07 & \\
\hline 92937_at & fibroblast growth factor receptor 4 & 2.03 & \\
\hline 160632_at & protein kinase $\mathrm{C}$ & 2.50 & \\
\hline 99521_at & adenylate kinase 3 alpha-like 1 & 3.16 & \\
\hline 99959_at & adenylate kinase 3 alpha-like 1 & 2.06 & \\
\hline 92492_at & adenylate kinase 3 & 2.48 & \\
\hline 101489_at & S-adenosylmethionine decarboxylase 1 & 2.14 & \\
\hline 100323_at & S-adenosylmethionine decarboxylase 1 & 2.19 & \\
\hline 102773_at & carbonic anhydrase 8 & 2.38 & \\
\hline 160375_at & carbonic anhydrase 3 & 3.80 & \\
\hline 103391_at & cysteine conjugate-beta lyase 2 & 3.11 & \\
\hline 160628_at & glycine C-acetyltransferase & 2.28 & \\
\hline \multicolumn{4}{|l|}{ Downregulated } \\
\hline 92898_at & cytochrome $\mathrm{P} 450$, family 7 , subfamily b, polypeptide 1 & 0.45 & \\
\hline 161345_f_at & cytochrome P450, family 7 , subfamily b, polypeptide 1 & 0.49 & \\
\hline 102192_r_at & acyl-CoA synthetase medium-chain family member 3 & 0.35 & \\
\hline Angiogenesis (59) & & & 1.00 \\
\hline \multicolumn{4}{|l|}{ Upregulated } \\
\hline 96038_at & ribonuclease, RNase A family 4 & 2.00 & \\
\hline 102373_at & glutamyl aminopeptidase & 2.20 & \\
\hline 102698_at & endothelial PAS domain protein 1 & 2.31 & \\
\hline Transport (814) & & & 0.510 \\
\hline \multicolumn{4}{|l|}{ Upregulated } \\
\hline 101913_at & chloride channel 5 & 2.32 & \\
\hline 102662_at & asialoglycoprotein receptor 2 & 2.70 & \\
\hline 103271_at & low density lipoprotein receptor-related protein 6 & 2.84 & \\
\hline
\end{tabular}


Table 2. Continued

\begin{tabular}{|c|c|c|c|}
\hline Affymetrix ID & Gene & KTE/Control ratio & EASE score \\
\hline 103958_g_at & transferrin receptor & 2.33 & \\
\hline 94792_at & macrophage scavenger receptor 1 & 2.37 & \\
\hline 96078_g_at & solute carrier family 17 (sodium phosphate), member 1 & 2.02 & \\
\hline 94433_at & solute carrier family 38, member 2 & 2.00 & \\
\hline 100491_at & solute carrier family 16 (monocarboxylic acid transporters), member 2 & 2.01 & \\
\hline 94797_at & solute carrier family 26 (sulfate transporter), member 1 & 2.26 & \\
\hline 96331_at & sorting nexin 2 & 2.08 & \\
\hline 97505_at & ADP-ribosylation factor-like 1 & 2.07 & \\
\hline 103703_f_at & similar to integral membrane transport protein UST1R & 2.91 & \\
\hline 92294_at & ADP-ribosylation factor-like $5 \mathrm{~A}$ & 2.28 & \\
\hline 93711_at & secretory protein SEC23 related gene & 2.22 & \\
\hline 94322_at & squalene epoxidase & 3.12 & \\
\hline 92840_at & nucleoporin 54 & 2.07 & \\
\hline 103619_at & cytochrome b5 type B & 2.38 & \\
\hline 96276_r_at & transmembrane 9 superfamily member 3 & 2.25 & \\
\hline 103702_i_at & similar to integral membrane transport protein UST1R & 2.36 & \\
\hline 96227_at & serine (or cysteine) peptidase inhibitor, clade A, member 6 & 3.94 & \\
\hline 98969_at & ATP-binding cassette, sub-family D (ALD), member 1 & 3.73 & \\
\hline 100491_at & solute carrier family 16 (monocarboxylic acid transporters), member 2 & 2.01 & \\
\hline 100951_at & polycystic kidney disease 2 & 2.15 & \\
\hline 103702_i_at & similar to integral membrane transport protein UST1R & 2.36 & \\
\hline 104421_at & flavin containing monooxygenase 3 & 4.67 & \\
\hline 92763_at & ATP-binding cassette, sub-family B (MDR/TAP), member 7 & 3.01 & \\
\hline \multicolumn{4}{|l|}{ Downregulated } \\
\hline 94642_at & guanosine diphosphate (GDP) dissociation inhibitor 2 & 0.30 & \\
\hline 100078_at & mouse apolipoprotein A-IV & 0.38 & \\
\hline Stress (39) & & & 0.403 \\
\hline Upregulated & & - & \\
\hline Downregulated & & - & \\
\hline Ribosomal (34) & & & 0.633 \\
\hline Upregulated & & - & \\
\hline Downregulated & & - & \\
\hline Others $(522)$ & & & 0.0825 \\
\hline \multicolumn{4}{|l|}{ Upregulated } \\
\hline 103076_at & chromatin modifying protein 5 & 2.30 & \\
\hline 102240_at & peroxisome proliferative activated receptor, gamma, coactivator 1 alpha & 2.52 & \\
\hline 94379_at & kinesin family member $1 \mathrm{~B}$ & 2.04 & \\
\hline 94325_at & 3-hydroxy-3-methylglutaryl-Coenzyme A synthase 1 & 2.34 & \\
\hline 101837_g_at & protein phosphatase $1 \mathrm{~B}$, magnesium dependent, beta isoform & 2.10 & \\
\hline 97778_at & ST3 beta-galactoside alpha-2,3-sialyltransferase 3 & 3.94 & \\
\hline 94818_at & O-linked N-acetylglucosamine (GlcNAc) transferase & 2.05 & \\
\hline 96623_at & UDP-glucose ceramide glucosyltransferase & 2.86 & \\
\hline 93575_at & gamma-glutamyl hydrolase & 2.46 & \\
\hline 98934_at & lethal, Chr 7, Rinchik 6 & 2.20 & \\
\hline 99669_at & lectin, galactose binding, soluble 1 & 2.14 & \\
\hline 93183_at & CDC91 cell division cycle 91-like 1 & 2.07 & \\
\hline 96775_at & chromobox homolog 1 & 2.23 & \\
\hline 99535_at & CCR4 carbon catabolite repression 4 -like & 3.79 & \\
\hline 96207_at & RNA binding motif, single stranded interacting protein 1 & 2.12 & \\
\hline 103944_at & RAD51-like 1 & 2.07 & \\
\hline 102225_at & RAB GTPase activating protein 1-like & 2.12 & \\
\hline 102696_s_at & phosphatidylinositol transfer protein, beta & 2.23 & \\
\hline 104202_at & transmembrane protein 1 & 2.50 & \\
\hline 93212_at & protein tyrosine phosphatase-like A domain containing 1 & 2.02 & \\
\hline 96572_at & 5-azacytidine induced gene 2 & 2.25 & \\
\hline 160135_at & nitrilase family, member 2 & 2.28 & \\
\hline 160365_at & eukaryotic translation initiation factor 2 , subunit 2 & 2.15 & \\
\hline 98349_at & interleukin 6 signal transducer & 2.02 & \\
\hline \multicolumn{4}{|l|}{ Downregulated } \\
\hline 97487_at & serine (or cysteine) peptidase inhibitor, clade E, member 2 & 0.46 & \\
\hline 102016_at & cell death-inducing DFFA-like effector c & 0.26 & \\
\hline 100431_at & leptin receptor & 0.47 & \\
\hline
\end{tabular}


Table 2. Continued

\begin{tabular}{|c|c|c|c|}
\hline Affymetrix ID & Gene & KTE/Control ratio & EASE score \\
\hline Unknown (1363) & & & 0.0797 \\
\hline \multicolumn{4}{|l|}{ Upregulated } \\
\hline 95050_at & cysteine and histidine-rich domain (CHORD)-containing, zinc-binding protei & 2.32 & \\
\hline 96258_at & microsomal glutathione S-transferase 3 & 5.30 & \\
\hline 96351_at & basic transcription factor 3 -like 4 & 2.06 & \\
\hline 98530_at & growth arrest specific 5 & 3.74 & \\
\hline 99649_at & glutamate cysteine ligase & 2.01 & \\
\hline 100042_at & hydroxyacyl glutathione hydrolase & 2.17 & \\
\hline 101966_s_at & ring finger protein 13 & 2.11 & \\
\hline 103871_at & sec23 interacting protein & 2.09 & \\
\hline 96215_f_at & uc53a11.r1 Mus musculus cDNA, 5 end & 3.00 & \\
\hline 97402_at & indolethylamine N-methyltransferase & 3.74 & \\
\hline 98428_at & spastin & 2.80 & \\
\hline 99988_at & dymeclin & 2.24 & \\
\hline 100949_at & cDNA sequence BC004044 & 2.25 & \\
\hline 101380_at & nudix (nucleoside diphosphate linked moiety X)-type motif 14 & 2.17 & \\
\hline 101393_at & annexin A3 & 2.32 & \\
\hline 101404_at & coiled-coil domain containing 115 & 2.16 & \\
\hline 103497_at & cDNA sequence $\mathrm{BC} 025546$ & 2.15 & \\
\hline 103519_at & carboxylesterase 1 & 2.54 & \\
\hline 103744_at & SH3 domain binding glutamic acid-rich protein like 2 & 2.32 & \\
\hline 103982_s_at & alcohol dehydrogenase 4 (class II), pi polypeptide & 2.57 & \\
\hline 103983_at & alcohol dehydrogenase 4 (class II), pi polypeptide & 3.32 & \\
\hline 103986_at & inhibin beta-C & 2.88 & \\
\hline 104398_at & tetraspanin 33 & 2.09 & \\
\hline 104640_f_at & josephin domain containing 3 & 2.08 & \\
\hline 104643_at & WW, C2 and coiled-coil domain containing 1 & 2.26 & \\
\hline 93646_at & twinfilin, actin-binding protein, homolog 1 & 2.22 & \\
\hline 94192_at & ganglioside-induced differentiation-associated-protein 10 & 3.30 & \\
\hline 96494_at & kelch-like 24 & 2.49 & \\
\hline 96518_at & WW, C2 and coiled-coil domain containing 1 & 2.19 & \\
\hline 101179_at & aspartate-glutamate-alanine-aspartate box polypeptide 6 & 2.44 & \\
\hline 160678_at & tetraspanin 12 & 2.35 & \\
\hline 160697_at & expressed sequence $\mathrm{C} 77080$ & 2.39 & \\
\hline 162058_f_at & AV271456 Mus musculus cDNA, 3 end & 3.11 & \\
\hline 160217_at & RIKEN cDNA 2310001A20 gene & 2.26 & \\
\hline 103403_at & RIKEN cDNA 2310007H09 gene & 3.18 & \\
\hline 98594_at & RIKEN cDNA 1190002 N15 gene & 2.58 & \\
\hline 102920_at & RIKEN cDNA 1810054D07 gene & 2.01 & \\
\hline \multicolumn{4}{|l|}{ Downregulated } \\
\hline X00686_M_at & mouse gene for $18 \mathrm{~S}$ rRNA. & 0.30 & \\
\hline 103460_at & DNA-damage-inducible transcript 4 & 0.43 & \\
\hline
\end{tabular}

Categories are gene ontology biological processes. EASE score is the Fisher Exact Statistic for the likelihood that the number of genes found in the filtered list occurred by chance sampling of the MG-U74A probe set population.

for liver weight and plasma parameters such as fasting and postprandial blood glucose levels and AST and ALT activities did not differ significantly between the two groups. Although KTE reduced the fasting blood glucose level in the type-2 diabetic model, ${ }^{5)}$ it did not reduce the fasting or the postprandial blood glucose level in normal mice.

DNA microarray analysis revealed that the expression of genes of known function, such as those for stress response, ribosomal proteins, transcription, cell function, the inflammatory/immune response, angiogenesis, transport, and metabolism (xenobiotic, glutathione, carbohydrate, lipid, catecholamine, etc.) remained largely unaffected by KTE. EASE analysis indicated that KTE did not have any selective effects on the expression of the genes associated with the above categories. Furthermore, the expression of oncogenes (alk, cyclin D1, erbA/ear, erB, fos, jun, myc, ras, and src) and antioncogenes (p16, p53, p107/p130, rb, and wt1) also was not modulated by KTE (data not shown).

The drug metabolism system comprises phase I and II enzymes. Phase I enzymes, mainly CYPs, detoxify a variety of endogenous and exogenous chemicals and activate many carcinogens. ${ }^{16)}$ The major CYPs involved in drug metabolism in the human body are hCYP1A2, hCYP2C9, hCYP2C19, hCYP2D6, hCYP2E1, and hCYP3A4. ${ }^{17)}$ Although there are more than 50 CYPs, $90 \%$ of drugs are metabolized by just six of these, the two most significant enzymes being hCYP3A4 and hCYP2D6, ${ }^{18)}$ but KTE upregulates the expression of mCYP2C70, whose human ortholog is hCYP2C9. In 
humans, hCYP2C9 is the rate-limiting enzyme in the metabolic clearance of clinically used drugs such as the hypoglycemic agents tolbutamide and glipizine, the anticonvulsant phenytoin, and the $S$-enantiomer of the anticoagulant warfarin. ${ }^{19,20)}$ KTE can influence the effects of these drugs, but it remains to be seen whether the changes in CYP2C70 mRNA expression induced by KTE are reflected as alterations in drug metabolism, since the induction level of the mRNA was not very high (2- to 5-fold). Further studies are necessary to determine the exact function of $\mathrm{mCYP} 2 \mathrm{C} 70$ in mice.

Phase II enzyme systems catalyze the conjugation of phase I metabolites to various water-soluble molecules such as glutathione or glucronic acid, thereby accelerating the metabolic excretion rate. Glutathione S-transferase (GST) is one of the most important phase II enzymes. In the present study, KTE slightly induced GSTs, suggesting that it modulated the drug-metabolizing capacity of the mice.

It has been reported that some functional foods, such as comfrey (Symphytum officinale), noni (Morinda citrifolia), and black cohosh (Cimicifuga racemosa), are hepatotoxic in experimental animals and humans. ${ }^{21-24)}$ Using DNA microarray analysis, Mei et al. founds that comfrey drastically regulates many CYP genes (e.g., CYP2A12, CYP4A12, CYP7A1, CYP2C12, and CYP26), GST genes (Gsta3, Gstm3, and Gstp1), ATP-binding cassette transporters (e.g., Abcb9 and Abcc3), and other metabolism-associated genes in the rat liver. ${ }^{21)}$ Although KTE altered the mRNA levels of drug-metabolizing enzymes by 2 - to 5 -fold in our study, comfrey was found to change them by as much as 20 -to 100 -fold. ${ }^{21,22)}$

In this study, there were no significant differences in AST or ALT activities between the KTE-treated and the control mice. Furthermore, hepatoprotective and antioxidative effects have been reported for hot-water extracts of the roots and stems of KT in liver injury model mice. ${ }^{25}$ Although KTE induced gene expression of some drug-metabolizing enzymes, it is probably not hepatotoxic in normal mice.

An anti-obesity effect has been reported for the aqueous extract of the roots of KT in Zucker fatty. ${ }^{26)}$ There are, however, only a few reports on the effect of KT consumption in normal mice. Kishino et al. have reported an antiobesity effect of a mixture of Salacia reticulata extract and cyclodextrin in normal C57BL/6J mice fed a high-fat diet. ${ }^{27)}$ The mechanisms of the antiobesity effect of KTE in normal mice has not yet been reported.

In this study, we used DNA microarray analysis and found that KTE regulated the mRNA level of genes related to energy metabolism, such as catechol-omethyltransferase, monoamine oxidase A, succinylCoA synthetase, 3-hydroxy-3-methylglutaryl-CoA reductase, and acyl-CoA synthetase medium-chain family member 3. This data suggests that KTE influences energy metabolism through gene regulation. Further research to clarify the mechanism of gene regulation by KTE is planned.

This study indicates that KTE has no significant acute hepatotoxicity. In conclusion, normal mice can safely consume KTE, and it might be effective as a functional food to maintain good health.

\section{Acknowledgments}

The authors wish to express their gratitude to Professor Keiko Abe and Associate Professor Ichiro Matsumoto, Graduate School of Agricultural and Life Sciences, The University of Tokyo, for their help in performing DNA microarray analyses.

\section{References}

1) Warrir, P. K., Nambir, V. P. K., and Ramankutty, C., Salacia reticulata wight. In "Indian Medical Plants," Orient Longman, Chennai, pp. 47-48 (1993).

2) Jayawardena, M. H., de Alwis, N. M., Hettigoda, V., and Fernando, D. J., A double blind randomized placebo controlled cross over study of a herbal preparation containing Salacia reticulata in the treatment of type 2 diabetes. J. Ethnopharmacol., 97, 215-218 (2005).

3) Karunanayake, E. H., Welihinda, J., Sirimanne, S. R., and Sinnadorai, G., Oral hypoglycaemic activity of some medicinal plants of Sri Lanka. J. Ethnopharmacol., 11, 223-231 (1984).

4) Kajimoto, O., Kawamori, S., Shimoda, H., Kawahara, Y., Hirata, H., and Takahashi, T., Effects of a diet containing Salacia reticulata on mild type 2 diabetes in humans. J. Jpn. Soc. Nutr. Food Sci., 53, 199-205 (2000).

5) Im, R., Mano, H., Matsuura, T., Nakatani, S., Shimizu, J., and Wada, M., Mechanisms of blood glucose-lowing effect of aqueous extract from stems of Kothala himburu (Salacia reticulata) in the mouse. J. Ethnopharmacol., in press.

6) Swinbanks, D., and O'Brien, J., Japan explores the boundary between food and medicine. Nature, 364, 180 (1993).

7) Arai, S., Morinaga, Y., Yoshikawa, T., Ichiishi, E., Kiso, Y., Yamazaki, M., Morotomi, M., Shimizu, M., Kuwata, $\mathrm{T}$., and Kaminogawa, S., Recent trends in functional food science and the industry in Japan. Biosci. Biotechnol. Biochem., 66, 2017-2029 (2002).

8) Shimoda, H., Furuhashi, T., Naitou, K., Nagase, T., and Okada, M., Thirteen-week repeat dose oral toxicity study of Salacia reticulata extract in rats. Jpn. J. Med. Pharm. Sci., 46, 527-541 (2001).

9) Arai, S., Studies on functional foods in Japan: state of the art. Biosci. Biotechnol. Biochem., 60, 9-15 (1996).

10) Muller, M., and Kersten, S., Nutrigenomics: goals and strategies. Nat. Rev. Genet., 4, 315-322 (2003).

11) Narasaka, S., Endo, Y., Fu, Z., Moriyama, M., Arai, S., Abe, K., and Kato, H., Safety evaluation of hypoallergenic wheat flour by using a DNA microarray. Biosci. Biotechnol. Biochem., 70, 1464-1470 (2006).

12) Kamakura, M., Maebuchi, M., Ozasa, S., Komori, M., Ogawa, T., Sakaki, T., and Moriyama, T., Influence of royal jelly on mouse hepatic gene expression and safety 
assessment with a DNA microarray. J. Nutr. Sci. Vitaminol., 51, 148-155 (2005).

13) Hosake, D. A., Dennis, G., Jr., Sherman, B. T., Lane, H. C., and Lempicki, R. A., Identifying biological themes within lists of genes with EASE. Genome Biol., 4, R70 (2003).

14) Dennis, G., Jr., Sherman, B. T., Hosack, D. A., Yang, J., Gao, W., Lane, H. C., and Lempicki, R. A., DAVID: database for annotation, visualization, and integrated discovery. Genome Biol., 4, P3 (2003).

15) Moller, D. E., New drug targets for type 2 diabetes and the metabolic syndrome. Nature, 414, 821-827 (2001).

16) Guengerich, F. P., and Shimada, T., Oxidation of toxic and carcinogenic chemicals by human cytochrome P-450 enzymes. Chem. Res. Toxicol., 4, 391-407 (1991).

17) Lynch, T., and Price, A., The effect of cytochrome $P 450$ metabolism on drug response, interactions, and adverse effects. Am. Fam. Physician., 76, 391-396 (2007).

18) Faber, M. S., Jetter, A., and Fuhr, U., Assessment of CYP1A2 activity in clinical practice: why, how, and when? Basic Clin. Pharmacol. Toxicol., 97, 125-134 (2005).

19) Miners, J. O., and Birkett, D. J., Cytochrome P4502C9: an enzyme of major importance in human drug metabolism. Br. J. Clin. Pharmacol., 45, 525-538 (1998).

20) Goldstein, J. A., Clinical relevance of genetic polymorphisms in the human CYP2C subfamily. Br. J. Clin. Pharmacol., 52, 349-355 (2001).

21) Mei, N., Guo, L., Zhang, L., Shi, L., Sun, Y. A., Fung, C., Moland, C. L., Dail, S. L., Fuscoe, J. C., and Chen,
T., Analysis of gene expression changes in relation to toxicity and tumorigrnrsis in the livers of Big Blue transgenic rats fed comfrey (Symphytum officinale). BMC Bioinformatics, 7, S16 (2006).

22) Gue, L., Mei, N., Dail, S., Fuscoe, J., and Chen, T., Comparison of gene expression profiles altered by comfrey and riddelliine in rat liver. BMC Bioinformatics, 8, S22 (2007).

23) Stadlbauer, V., Fickert, P., Lackner, C., Schmerlaib, J., Krisper, P., Trauner, M., and Stauber, R. E., Hepatotoxicity of NONI juice: report of two cases. World $J$. Gastroenterol., 14, 4758-4760 (2005).

24) Vitetta, L., Thomsen, M., and Sali, A., Black cohosh and other herbal remedies associated with acute hepatitis. Med. J. Australia, 180, 598-600 (2004).

25) Yoshikawa, M., Ninomiya, K., Shimoda, H., Nishida, N., and Matsuda, H., Hepatoprotective and antioxidative properties of Salacia reticulata: preventive effects of phenolic constituents on $\mathrm{CCl}_{4}$-induced liver injury in mice. Biol. Pharm. Bull., 25, 72-76 (2002).

26) Yoshikawa, M., Shimoda, H., Nishida, N., Takada, M., and Matsuda, H., Salacia reticulata and its polyphenolic constituents with lipase inhibitory and lipolytic activities have mild antiobesity effects in rats. J. Nutr., 132, 18191824 (2002).

27) Kishino, E., Ito, T., Fujita, K., and Kiuchi, Y., A mixture of the Salacia reticulata (Kotala himbutu) aqueous extract and cyclodextrin reduces the accumulation of visceral fat mass in mice and rats with high-fat dietinduced obesity. J. Nutr., 19, 433-439 (2007). 